\title{
Efficacy of teaching methods used to develop critical thinking in nursing and midwifery undergraduate students: A systematic review of the literature.
}

\begin{abstract}
Background: The value and importance of incorporating strategies that promote critical thinking in nursing and midwifery undergraduate programs is well documented. However, relatively little is known about the effectiveness of teaching strategies in promoting CT. Evaluating effectiveness is important to promote 'best practice' in teaching.
\end{abstract}

Objective: To evaluate the efficacy of teaching methods used to develop critical thinking skills in nursing and midwifery undergraduate students.

Data sources: The following six databases; CINAHL, Ovid Medline, ERIC, Informit, PsycINFO and Scopus were searched and resulted in the retrieval of 1,315 papers.

Review methods: After screening for inclusion, each paper was evaluated using the Critical Appraisal Skills Programme tool. Twenty-eight studies met the inclusion criteria and quality appraisal.

Results: Twelve different teaching interventions were tested in 8 countries. Results varied, with little consistency across studies using the same type of intervention or outcome tool. Sixteen tools were used to measure the efficacy of teaching in developing critical thinking. Seventeen studies identified a significant increase in critical thinking, while nine studies found no increases, and two found unexplained decreases in CT when using a similar educational intervention.

Conclusions: Whilst this review aimed to identify effective teaching strategies that promote and develop critical thinking, flaws in methodology and outcome measures contributed to inconsistent findings. The continued use of generalised CT tools is unlikely to help identify appropriate teaching methods that will improve CT abilities of midwifery and nursing students and prepare them for practice. The review was limited to empirical studies published in English that used measures of critical thinking with midwifery and nursing students. Discipline specific strategies and tools that measure students' abilities to apply CT in practice are needed.

Key words: critical thinking, nursing, midwifery, teaching methods, evaluation

\section{Highlights}

- Evidence-based teaching methods are needed to develop critical thinking skills.

- Evaluation of teaching methods and effect on critical thinking is inconsistent.

- Problem-based learning methods are commonly used to develop critical thinking. 
- Standardised, discipline specific tools are required to measure critical thinking in practice.

- Active constructivist-based learning strategies are vital in educating nurses.

\section{Introduction}

Critical thinking (CT) involves making judicious purposeful judgements as a result of engaging in a process of analysis, interpretation evaluation, inference, explanation, and reflection (Facione, 1990). According to Castledine (2010), critical thinking requires clinicians to carefully define and analyse problems, with a sense of inquisitiveness and questioning of information and decisions. This sense of inquiry is crucial for nurses and midwives working in complex and demanding environments with increased accountability, autonomy and collaboration with other disciplines (Muoni, 2012; Pucer et al., 2014; Castledine, 2010). Therefore, an important aim of nursing and midwifery undergraduate education is to develop students' critical thinking abilities in preparation for practice.

Although there is agreement about the value and importance of incorporating strategies that promote critical thinking in nursing and midwifery undergraduate programs there is little understanding regarding the best approaches to develop these skills (Tiwari, et al, 2006). The inadequacy of the traditional lecture format to promote critical thinking is well documented (Banfield, et al 2012; Popil, 2011). However, relatively little is known about the effectiveness of active learning strategies in promoting critical thinking.

A qualitative systematic review of critical thinking development in nursing explored participant's perspectives, as well as facilitators and barriers (Chan, 2013). Analysis of the 17 studies illustrated that the definition and concept of critical thinking changed from time to time, and identified the need to clarify educators' perspectives towards critical thinking. This review did not include any quantitative studies and tools used to measure the impact of teaching strategies on critical thinking development were not reported. Accordingly, Chan (2013) recommended an evaluation of teaching strategies designed to develop critical thinking skills be undertaken.

Evaluating the effectiveness of teaching strategies and their impact on critical thinking is important to promote 'best practice'. The purpose of the current systematic review was to determine the efficacy of teaching methods used to develop critical thinking in nursing and midwifery undergraduate students.

\section{Search Strategies Utilised}

A search of major databases CINAHL, Ovid Medline, ERIC, Informit, PsycINFO and Scopus, was conducted in October 2015. The search criteria was limited to articles published in English and within 
peer reviewed journals for the period 2001- 2015. This timeframe was identified to build on from the publication by Scheffer and Rubenfeld (2000) who used a Delphi study to develop a consensus definition of $\mathrm{CT}$ in nursing. It was considered that scholarly research in this area would have occurred following this seminal work.

The inclusion criteria were original research studies that utilised an experimental design to assess CT development following a specific educational intervention in undergraduate nursing and/or midwifery. Papers were excluded if critical thinking was not specifically measured more than once, did not test a specific educational strategy, the sample was post-graduate students, full text was not available in English, discussion papers that did not involve original research study, or did not use an experimental design.

Five search terms were entered into the databases with the article title, abstract and body searched. The search terms used were:

1. "critical thinking" AND midwife*

2. "critical thinking" AND midwife* AND measure*

3. "critical thinking" AND midwife* AND evaluat*

4. "critical thinking" AND students, nursing AND measure*

5. "critical thinking" AND students, nursing AND evaluat*

The search was conducted sequentially using the six databases and search terms. An initial search, filtering for date, language and source of publication, identified 1,315 papers. Following the guidelines suggested by Kable et al. (2012), once duplicates were excluded, each identified citation was reviewed and filtered through three screening levels; (i) title; (ii) title and abstract; and (iii) fulltext. Articles that were not relevant or did not meet inclusion criteria were discarded. Twenty-nine papers were included. No papers involving midwifery undergraduate students met the inclusion criteria, therefore the samples of all included studies were undergraduate nursing students.

Each paper was assessed for relevance by reading the abstract (and where necessary the entire paper) using the inclusion and exclusion criteria to assess relevance to this review. Articles that met the inclusion criteria were listed in a summary table (Table 1) during the search. After the initial search all articles identified in subsequent searches were checked against articles in the summary table and duplicates excluded. Each article was also entered into a reference management database (Endnote) including the search term and engine used to locate each article. A quality appraisal process was performed using the Critical Appraisal Skills Programme (CASP) tool (CASP, 2013) and 
one article of poor quality was excluded, the excluded study was listed in the summary table. Following the quality appraisal process 28 papers were selected for review.

\section{Results}

All 28 included studies involved the measurement of critical thinking skill development or change following completion of a specific educational intervention. The most common educational interventions were problem-based learning (PBL) (7 studies), simulation (6 studies), concept mapping (4 studies), and a combination of PBL and concept mapping (2 studies). The remaining 9 studies examined a diverse range of teaching interventions.

A variety of tools $(n=16)$ were used to measure critical thinking development. Sixteen $(57 \%)$ of the 28 studies utilised one of three standardised commercially available tools to measure critical thinking. These were the California Critical Thinking Disposition Inventory (CCTDI) (8 studies), the California Critical Thinking Skills Test (CCTST) (3 studies), and Health Services Reasoning Test (HSRT) (3 studies). Two studies used both the Californian Critical Thinking Skills Test and California Critical Thinking Disposition Inventory. A previous systematic review of tools used to measure critical thinking found limited reporting of the reliability of these three tools, little emphasis placed on establishing validity of newly developed tools, and inconsistent results across studies using standardised tools (Carter et al., 2015). Although the results of the studies in this current review could be affected by the reliability and validity of the outcome measures, the focus of this systematic review is to establish the efficacy of different teaching methods in critical thinking development.

Most studies were conducted in the USA $(n=13)$. There was an increasing number of studies from countries where traditional lecture style teaching formats have predominated such as Taiwan $(n=4)$, Korea ( $n=3)$, China ( $n=2)$, Hong Kong $(n=2)$, Iran (2), Turkey $(n=1)$, and Slovenia $(n=1)$. The results of the papers reviewed will be outlined below and grouped according to the specific teaching strategy utilised. 
Title: Efficacy of teaching methods used to develop critical thinking in nursing and midwifery undergraduate students: A systematic review of the literature

Table 1: Articles that met inclusion and quality criteria

\begin{tabular}{|c|c|c|c|c|c|c|c|}
\hline No & $\begin{array}{l}\text { Author, year } \\
\text { and country }\end{array}$ & $\begin{array}{l}\text { Teaching } \\
\text { intervention }\end{array}$ & Participants & $\begin{array}{l}\text { Measurement tool } \\
\text { Time between pre-post } \\
\text { test interventions }\end{array}$ & Results & Limitations & $\begin{array}{l}\text { Quality } \\
\text { Appraisal } \\
\text { using CASP }\end{array}$ \\
\hline \multicolumn{8}{|c|}{ Problem Based Learning (PBL) } \\
\hline 1 & $\begin{array}{l}\text { Choi et al. } \\
(2014) . \\
\text { Korea }\end{array}$ & $\begin{array}{l}16 \text { week program } \\
\text { of } \mathrm{PBL} \text { compared } \\
\text { to traditional } \\
\text { lecture }\end{array}$ & $\begin{array}{l}1^{\text {st }} \text { year nursing } \\
\text { students }(\mathrm{n}=90) \\
\text { recruited from } 2 \\
\text { colleges. Students } \\
\text { from college A } \\
\text { received PBL and } \\
\text { students from } \\
\text { college B received } \\
\text { lectures. }\end{array}$ & $\begin{array}{l}\text { Critical Thinking Ability } \\
\text { Scale (CTAS) for } \\
\text { College Students used } \\
\text { at baseline and } 16 \\
\text { weeks following } \\
\text { instruction. }\end{array}$ & $\begin{array}{l}\text { No significant differences } \\
\text { in critical thinking scores } \\
\text { between PBL and } \\
\text { traditional lecture groups. }\end{array}$ & $\begin{array}{l}\text { Students recruited } \\
\text { from two different } \\
\text { colleges and may have } \\
\text { differed in academic } \\
\text { ability. } \\
\text { Small sample } \\
\text { underpowered study. }\end{array}$ & Include \\
\hline 2 & $\begin{array}{l}\text { Jun et al. } \\
\text { (2013). } \\
\text { South Korea }\end{array}$ & $\begin{array}{l}\text { Intervention group } \\
\text { experienced the } \\
5 E \text { learning cycle } \\
\text { model with PBL } \\
\text { for five weeks. } \\
\text { Control group } \\
\text { received lecture } \\
\text { and practice. }\end{array}$ & $\begin{array}{l}1^{\text {st }} \text { year nursing } \\
\text { students }(n=161)\end{array}$ & $\begin{array}{l}\text { Critical Thinking } \\
\text { Disposition Scale for } \\
\text { Nursing Students } \\
\text { (CTDS). Pre and post } \\
\text { tests performed } 4 \text { weeks } \\
\text { apart }\end{array}$ & $\begin{array}{l}\text { Statistically significant } \\
\text { increase in critical thinking } \\
\text { scores for experimental } \\
\text { group. }\end{array}$ & $\begin{array}{l}\text { CTDS not available in } \\
\text { English, } 20 \text { point self } \\
\text { report Likert scale } \\
\text { measures disposition } \\
\text { as a proxy for critical } \\
\text { thinking skills. The } \\
\text { education intervention } \\
\text { was brief, limiting its' } \\
\text { impact. }\end{array}$ & Include \\
\hline 3 & $\begin{array}{l}\text { Tiwari et al. } \\
(2006) \text {. Hong } \\
\text { Kong }\end{array}$ & $\begin{array}{l}\text { Intervention was a } \\
12 \text { month PBL } \\
\text { program. Control } \\
\text { group had } \\
\text { traditional lectures }\end{array}$ & $\begin{array}{l}1^{\text {st }} \text { year nursing } \\
\text { students }(n=79)\end{array}$ & $\begin{array}{l}\text { Californian Critical } \\
\text { Thinking Disposition } \\
\text { Inventory (CCTDI), at } 4 \\
\text { time points, pretest, end } \\
\text { of } 1 \text { st, } 2^{\text {nd }} \text { and } 3 \text { rd years. } \\
\text { Qualitative comments } \\
\text { were also collected from } \\
\text { students. }\end{array}$ & $\begin{array}{l}\text { Significantly greater } \\
\text { improvement in critical } \\
\text { thinking scores for } \\
\text { experimental group on } \\
\text { completion of course. } \\
\text { Scores still significantly } \\
\text { higher after } 2 \text { years, } \\
\text { although lower than }\end{array}$ & $\begin{array}{l}\text { PBL conducted for one } \\
\text { year, perhaps greater } \\
\text { differences could have } \\
\text { been achieved if } \\
\text { continued throughout } \\
\text { degree. Could be } \\
\text { argued that } 1 \text { year was } \\
\text { enough to bring about } \\
\text { change }\end{array}$ & Include \\
\hline
\end{tabular}




\begin{tabular}{|c|c|c|c|c|c|c|c|}
\hline & & & & & $\begin{array}{l}\text { immediately following the } \\
\text { program. }\end{array}$ & $\begin{array}{l}\text { Measured critical } \\
\text { thinking disposition } \\
\text { rather than skill. }\end{array}$ & \\
\hline 4 & $\begin{array}{l}\text { Jones, } \\
\text { (2008). USA }\end{array}$ & $\begin{array}{l}\text { Intervention of } \\
\text { traditional } \\
\text { teaching for two } \\
\text { weeks and then } \\
\text { weekly PBL } \\
\text { sessions. Control } \\
\text { group received } \\
\text { traditional } \\
\text { teaching involving } \\
\text { pre and post } \\
\text { conference } \\
\text { lectures. }\end{array}$ & $\begin{array}{l}\text { 2nd year nursing } \\
\text { students }(n=60) \text {. }\end{array}$ & $\begin{array}{l}\text { Critical Thinking } \\
\text { measured by grading } \\
\text { students' written care } \\
\text { plans based on } 6 \text { levels } \\
\text { of Blooms taxonomy of } \\
\text { cognitive learning. }\end{array}$ & $\begin{array}{l}\text { Intervention group } \\
\text { demonstrated higher } \\
\text { critical thinking scores } \\
\text { compared to control } \\
\text { group. }\end{array}$ & $\begin{array}{l}\text { Potential bias as } \\
\text { principal investigator } \\
\text { taught both groups, } \\
\text { evaluated their work, } \\
\text { and was not blinded to } \\
\text { the intervention }\end{array}$ & Include \\
\hline 5 & $\begin{array}{l}\text { Yu et al. } \\
\text { (2012). } \\
\text { China }\end{array}$ & $\begin{array}{l}\text { Intervention } \\
\text { consisted of PBL, } \\
\text { control group } \\
\text { received lecture } \\
\text { based learning. } \\
\text { Timeframe of } \\
\text { these approaches } \\
\text { was not stated. }\end{array}$ & $\begin{array}{l}2^{\text {nd }} \text { year nursing } \\
\text { students }(n=76)\end{array}$ & $\begin{array}{l}\text { Chinese Version of } \\
\text { Californian Critical } \\
\text { Thinking Disposition } \\
\text { Inventory (CCTDI) } \\
\text { administered before, } \\
\text { after the first learning } \\
\text { process (timeframe not } \\
\text { stated) and after the } \\
\text { semester-long course. }\end{array}$ & $\begin{array}{l}\text { Statistical improvement in } \\
\text { overall CTDI scores } \\
\text { following PBL. However, } \\
\text { PBL students' critical } \\
\text { thinking disposition scores } \\
\text { did not show improvement } \\
\text { on analyticity, } \\
\text { systematicity, and critical } \\
\text { thinking self-confidence } \\
\text { subscale scores. }\end{array}$ & $\begin{array}{l}\text { The subscales of Truth } \\
\text { seeking, systematicity, } \\
\text { and self-confidence } \\
\text { scored below the cut- } \\
\text { off of } 40 \text {, this may be } \\
\text { explained by cultural } \\
\text { approaches to learning } \\
\text { which do not } \\
\text { encourage critical } \\
\text { thinking } \\
\text { Potential } \\
\text { contamination of } \\
\text { results if students } \\
\text { shared learning } \\
\text { experiences }\end{array}$ & Include \\
\hline 6 & $\begin{array}{l}\text { Dehkordi and } \\
\text { Heydarnejad, } \\
\text { (2008). Iran }\end{array}$ & $\begin{array}{l}\text { PBL for a one } \\
\text { semester course. } \\
\text { Control group } \\
\text { received } \\
\text { traditional } \\
\text { lectures. }\end{array}$ & $\begin{array}{l}2^{\text {nd }} \text { year nursing } \\
\text { students }(n=40)\end{array}$ & $\begin{array}{l}\text { Californian Critical } \\
\text { Thinking Disposition } \\
\text { Inventory (CCTDI) given } \\
\text { prior to and following the } \\
\text { semester. }\end{array}$ & $\begin{array}{l}\text { Statistical improvement in } \\
\text { CTDI scores following } \\
\text { PBL. }\end{array}$ & $\begin{array}{l}\text { Students may have } \\
\text { had limited previous } \\
\text { exposure to any active } \\
\text { teaching strategies } \\
\text { and therefore } \\
\text { responded positively to } \\
\text { PBL. }\end{array}$ & Include \\
\hline
\end{tabular}




\begin{tabular}{|c|c|c|c|c|c|c|c|}
\hline 7 & $\begin{array}{l}\text { Yuan et al. } \\
(2008) . \\
\text { China }\end{array}$ & $\begin{array}{l}\text { Intervention } \\
\text { received PBL ( } 36 \\
\text { learning hours, } \\
2 \text { hrs x } 18 \text { weeks) } \\
\text { Control received } \\
\text { lectures. }\end{array}$ & $\begin{array}{l}2^{\text {nd }} \text { year nursing } \\
\text { students }(n=46)\end{array}$ & $\begin{array}{l}\text { California Critical } \\
\text { Thinking Skills Test } \\
\text { (CCTST) Chinese- } \\
\text { Taiwan Version used at } \\
\text { baseline and end of } \\
\text { semester. }\end{array}$ & $\begin{array}{l}\text { PBL students had } \\
\text { significantly greater } \\
\text { improvements on overall } \\
\text { CCTST. }\end{array}$ & $\begin{array}{l}\text { Small sample. } \\
\text { Involved a single PBL } \\
\text { course embedded in a } \\
\text { traditional non-PBL } \\
\text { curriculum, which } \\
\text { might hinder the } \\
\text { development of } \\
\text { students' critical } \\
\text { thinking over time. } \\
\text { Potential } \\
\text { contamination of } \\
\text { results if students } \\
\text { shared learning } \\
\text { experiences }\end{array}$ & Include \\
\hline \multicolumn{8}{|c|}{ Concept Mapping } \\
\hline 8 & $\begin{array}{l}\text { Atay and } \\
\text { Karabacak } \\
\text { (2012). } \\
\text { Turkey }\end{array}$ & $\begin{array}{l}\text { Intervention was } 3 \\
\text { x 3-4 hour } \\
\text { education } \\
\text { sessions on } \\
\text { preparing concept } \\
\text { map care plans. } \\
\text { Control group } \\
\text { prepared care } \\
\text { plans using the } \\
\text { column format. }\end{array}$ & $\begin{array}{l}80 \text { freshman and } \\
\text { sophomore nursing } \\
\text { students. }\end{array}$ & $\begin{array}{l}\text { Used Californian Critical } \\
\text { Thinking Disposition } \\
\text { Inventory (CCTDI). } \\
\text { Timeframe between pre- } \\
\text { post test not stated. }\end{array}$ & $\begin{array}{l}\text { Statistically significant } \\
\text { increase in CT scores for } \\
\text { experimental group. }\end{array}$ & $\begin{array}{l}\text { Timeframe between } \\
\text { pre-post test not } \\
\text { stated. Intervention } \\
\text { group received an } \\
\text { extra } 9-12 \text { hours of } \\
\text { education compared to } \\
\text { control group. }\end{array}$ & Include \\
\hline 9 & $\begin{array}{l}\text { Wheeler and } \\
\text { Collins, } \\
\text { (2003) } \\
\text { United States }\end{array}$ & $\begin{array}{l}\text { Intervention } \\
\text { involved concept } \\
\text { mapping of } \\
\text { patient } \\
\text { information. } \\
\text { Control group } \\
\text { taught to use } \\
\text { traditional nursing } \\
\text { care plans. }\end{array}$ & $\begin{array}{l}\text { A convenience } \\
\text { sample }(n=76) \\
\text { was randomly } \\
\text { assigned to } \\
\text { experimental }(n= \\
\text { 44) and control }(n= \\
\text { 32) groups. }\end{array}$ & $\begin{array}{l}\text { California Critical } \\
\text { Thinking Skills Test } \\
\text { (CCTST) given between } \\
\text { pre-post tests ( } 7.5 \text { week } \\
\text { timeframe). }\end{array}$ & $\begin{array}{l}\text { Significant difference } \\
\text { between pre - post test } \\
\text { scores for both groups. } \\
\text { No difference found } \\
\text { between experimental } \\
\text { and control groups. }\end{array}$ & $\begin{array}{l}\text { Students exposed to } \\
\text { concept mapping for } \\
7.5 \text { weeks which may } \\
\text { be insufficient. Only } \\
1 / 3 \text { of students in one } \\
\text { course prepared } \\
\text { concept maps. } \\
\text { Possible } \\
\text { contamination of the } \\
2 / 3 \text { who did not } \\
\text { complete a concept } \\
\text { map }\end{array}$ & Include \\
\hline
\end{tabular}




\begin{tabular}{|c|c|c|c|c|c|c|c|}
\hline 10 & $\begin{array}{l}\text { Lee et al. } \\
(2013) \\
\text { Taiwan }\end{array}$ & $\begin{array}{l}\text { Intervention } \\
\text { concept map } \\
\text { teaching over } 15 \\
\text { weeks. Control } \\
\text { received } \\
\text { traditional } \\
\text { lectures. }\end{array}$ & 95 students. & $\begin{array}{l}\text { Used Critical Thinking } \\
\text { Scale (CTS) at } 4 \text { points } \\
\text { (beginning of } 1^{\text {st }} \\
\text { semester, before the } \\
\text { intervention, after the } \\
\text { intervention and before } \\
\text { graduation). }\end{array}$ & $\begin{array}{l}\text { Both control and } \\
\text { experimental groups had } \\
\text { higher initial critical } \\
\text { thinking scores that } \\
\text { tended to decrease over } \\
\text { time. }\end{array}$ & $\begin{array}{l}\text { The intervention was } \\
\text { only one semester, } \\
\text { then teaching reverted } \\
\text { to lecturer format. Yet } \\
\text { Critical thinking scores } \\
\text { were measured for } \\
\text { duration of program. }\end{array}$ & \\
\hline 11 & $\begin{array}{l}\text { Abel. and } \\
\text { Freeze, } \\
\text { (2006) USA }\end{array}$ & $\begin{array}{l}\text { Intervention } \\
\text { involved student } \\
\text { developing } 4 \\
\text { concept maps } \\
\text { over } 4 \text { semesters. } \\
\text { No control group. }\end{array}$ & $\begin{array}{l}28 \text { associate } \\
\text { degree nursing } \\
\text { students. }\end{array}$ & $\begin{array}{l}\text { Used concept map } \\
\text { scoring for each of the } 4 \\
\text { concept maps } \\
\text { completed. }\end{array}$ & $\begin{array}{l}\text { There was a significant } \\
\text { increase in mean scores } \\
\text { of the first concept map to } \\
\text { the average mean score } \\
\text { of the last two maps } \\
(p=0.05) \text {. }\end{array}$ & $\begin{array}{l}\text { Tool measured } \\
\text { competence in using a } \\
\text { concept map rather } \\
\text { than critical thinking. } \\
\text { No relationship } \\
\text { between measurement } \\
\text { tool and critical } \\
\text { thinking. }\end{array}$ & Include \\
\hline \multicolumn{8}{|c|}{ Concept Mapping and PBL } \\
\hline 12 & $\begin{array}{l}\text { Tseng et al. } \\
(2011) . \\
\text { Taiwan }\end{array}$ & $\begin{array}{l}\text { Intervention was } \\
3 \text { hrs of PBL for } 14 \\
\text { weeks and } 42 \\
\text { hours of scenario } \\
\text { and discussion of } \\
\text { concept mapping. } \\
\text { Control group } \\
\text { received } \\
\text { traditional lecture } \\
\text { based teaching }\end{array}$ & 120 RN students. & $\begin{array}{l}\text { 10-item Critical-Thinking } \\
\text { Scale (CTS) used } \\
\text { before the course began } \\
\text { (pre-test), at the end of } \\
\text { the course (post-test), } \\
\text { and six months after the } \\
\text { course (follow-up). }\end{array}$ & $\begin{array}{l}\text { CTS scores were } \\
\text { significantly higher in the } \\
\text { experimental group at } \\
\text { post-test and follow-up. }\end{array}$ & $\begin{array}{l}\text { Promising results } \\
\text { regarding retention of } \\
\text { higher critical thinking } \\
\text { scores following } \\
\text { graduation. } \\
\text { Potential } \\
\text { contamination from } \\
\text { students talking to } \\
\text { each other about PBL }\end{array}$ & Include \\
\hline 13 & $\begin{array}{l}\text { Orique and } \\
\text { McCarthy } \\
(2015) \\
\text { USA }\end{array}$ & $\begin{array}{l}\text { Intervention } 2 \\
\text { sessions of PBL } \\
\text { instruction and } 1 \\
\text { session of } \\
\text { concept mapping } \\
\text { in relation to the } \\
\text { development of } \\
\text { nursing care } \\
\text { plans. }\end{array}$ & $\begin{array}{l}1^{\text {st }} \text { year nursing } \\
\text { students }(n=49)\end{array}$ & $\begin{array}{l}\text { Holistic Critical Thinking } \\
\text { Rubric (HCTR) } \\
\text { measured critical } \\
\text { thinking in nursing care } \\
\text { plans. Students } \\
\text { submitted } 4 \text { nursing care } \\
\text { plans, prior to PBL or } \\
\text { concept map teaching, } \\
\text { following PBL, following } \\
\text { concept mapping and } \\
\text { then finally following }\end{array}$ & $\begin{array}{l}\text { There was a significant } \\
\text { increase in critical thinking } \\
\text { scores across the four } \\
\text { nursing care plans } \\
\text { submitted. }\end{array}$ & $\begin{array}{l}\text { Results may reflect } \\
\text { students' increasing } \\
\text { expertise in care } \\
\text { planning rather than } \\
\text { critical thinking per se. } \\
\text { No reporting of inter- } \\
\text { rater reliability of the } \\
\text { tool. No description of } \\
\text { assessment process, } \\
\text { and who completed } \\
\text { ratings. }\end{array}$ & Include \\
\hline
\end{tabular}




\begin{tabular}{|c|c|c|c|c|c|c|c|}
\hline & & & & $\begin{array}{l}\text { both teaching } \\
\text { methodologies. }\end{array}$ & & & \\
\hline \multicolumn{8}{|c|}{ Simulation } \\
\hline 14 & $\begin{array}{l}\text { Sullivan- } \\
\text { Mann et al. } \\
\text { (2009). } \\
\text { United States }\end{array}$ & $\begin{array}{l}\text { Controls received } \\
\text { two simulation } \\
\text { scenarios. } \\
\text { Students in the } \\
\text { intervention } \\
\text { received five } \\
\text { scenarios. }\end{array}$ & $\begin{array}{l}\text { Associate degree } \\
\text { nursing students } \\
(n=53)\end{array}$ & $\begin{array}{l}\text { Health Sciences } \\
\text { Reasoning Tool (HSRT). } \\
6 \text { week period between } \\
\text { pre and post-test. }\end{array}$ & $\begin{array}{l}\text { Statistically significant } \\
\text { increase in critical thinking } \\
\text { scores for experimental } \\
\text { group. }\end{array}$ & $\begin{array}{l}\text { Small sample size, } \\
\text { different facilitators for } \\
\text { the groups with varied } \\
\text { levels of experience. } \\
\text { Unclear if controls } \\
\text { received any } \\
\text { instruction in lieu of the } \\
\text { remaining } 3 \text { sessions. }\end{array}$ & Include \\
\hline 15 & $\begin{array}{l}\text { Ravert, } \\
\text { (2008). } \\
\text { United States }\end{array}$ & $\begin{array}{l}\text { Two experimental } \\
\text { groups } \\
\text { (1) } 5 \text { sessions in } \\
\text { non Human } \\
\text { Patient Simulation } \\
\text { (HPS) + } 5 \\
\text { discussion group } \\
\text { sessions + } \\
\text { education } \\
\text { sessions } \\
\text { (2) HPS group -5 } \\
\text { patient simulation } \\
\text { + education } \\
\text { sessions. } \\
\text { Control group } \\
\text { attended } \\
\text { education } \\
\text { sessions only. }\end{array}$ & $\begin{array}{l}1^{\text {st }} \text { nursing students } \\
(n=30)\end{array}$ & $\begin{array}{l}\text { Californian Critical } \\
\text { Thinking Skills Test } \\
\text { (CCTST) and } \\
\text { Californian Critical } \\
\text { Thinking Disposition } \\
\text { Inventory (CCTDI). One } \\
\text { semester between pre } \\
\text { and post- test. }\end{array}$ & $\begin{array}{l}\text { No differences in critical } \\
\text { thinking scores between } \\
\text { groups. }\end{array}$ & $\begin{array}{l}\text { Small sample size may } \\
\text { limit statistical } \\
\text { differences. The } \\
\text { critical thinking } \\
\text { instruments do not } \\
\text { measure concepts } \\
\text { related to discipline- } \\
\text { specific content. } \\
\text { Disparity between } \\
\text { intervention vs control } \\
\text { dose. Potential } \\
\text { contamination from } \\
\text { students talking to } \\
\text { each other about HPS } \\
\text { and what was learnt. }\end{array}$ & Include \\
\hline 16 & $\begin{array}{l}\text { Shinnick. and } \\
\text { Woo, (2013). } \\
\text { United States }\end{array}$ & $\begin{array}{l}\text { Intervention } \\
\text { consisted of a } \\
\text { single Human } \\
\text { Patient Simulation } \\
\text { session. No } \\
\text { control group } \\
\text { used. }\end{array}$ & $\begin{array}{l}3^{\text {rd }} \text { and } 4^{\text {th }} \text { year } \\
\text { nursing students } \\
(n=154) \text { from three } \\
\text { Schools of Nursing. }\end{array}$ & $\begin{array}{l}\text { Used Health Sciences } \\
\text { Reasoning Tool (HSRT) } \\
\text { at baseline and two } \\
\text { weeks after a single } \\
\text { Human Patient } \\
\text { Simulation. }\end{array}$ & $\begin{array}{l}\text { Following HPS there were } \\
\text { no statistically significant } \\
\text { gains in critical thinking. } \\
\text { There was a decrease in } \\
\text { scores (not statistically } \\
\text { significant). }\end{array}$ & $\begin{array}{l}\text { Very short intervention } \\
\text { and assessment } \\
\text { timeframe and hence } \\
\text { limited ability to impact } \\
\text { critical thinking. }\end{array}$ & Include \\
\hline
\end{tabular}




\begin{tabular}{|c|c|c|c|c|c|c|c|}
\hline 17 & $\begin{array}{l}\text { Wood and } \\
\text { Toronto } \\
\text { (2012) USA }\end{array}$ & $\begin{array}{l}\text { Intervention group } \\
\text { practised critical } \\
\text { assessment skills } \\
\text { for } 2 \text { hours using } \\
\text { HPS plus } \\
\text { traditional practice } \\
\text { (out of class } \\
\text { practice with } \\
\text { peers). Control } \\
\text { group used } \\
\text { traditional practice } \\
\text { only. }\end{array}$ & $\begin{array}{l}2^{\text {nd }} \text { year nursing } \\
\text { students }(n=85)\end{array}$ & $\begin{array}{l}\text { Californian Critical } \\
\text { Thinking Disposition } \\
\text { Inventory administered } 2 \\
\text { weeks prior to and } \\
\text { following intervention. }\end{array}$ & $\begin{array}{l}\text { Higher mean post-test } \\
\text { total scores compared } \\
\text { with pre-test total scores } \\
\text { in experimental group } \\
\text { students. }\end{array}$ & $\begin{array}{l}\text { The intervention group } \\
\text { received an extra two } \\
\text { hours of education } \\
\text { than the control group. } \\
\text { Measured critical } \\
\text { thinking disposition } \\
\text { rather than skill. }\end{array}$ & Include \\
\hline 18 & $\begin{array}{l}\text { Goodstone et } \\
\text { al. (2013). } \\
\text { USA }\end{array}$ & $\begin{array}{l}\text { Intervention high } \\
\text { fidelity patient } \\
\text { simulation (HFPS) } \\
\text { vs Control } \\
\text { consisted of } \\
\text { paper-and-pencil } \\
\text { case study group } \\
\text { work. }\end{array}$ & $\begin{array}{l}1^{\text {st }} \text { semester } \\
\text { associate degree } \\
\text { nursing students } \\
(n=42) .\end{array}$ & $\begin{array}{l}\text { Health Sciences } \\
\text { Reasoning Tool (HSRT) } \\
\text { used at week } 2 \text { and } \\
\text { week } 14 .\end{array}$ & $\begin{array}{l}\text { There was a significant } \\
\text { increase in the HSRT } \\
\text { scores for the case study } \\
\text { group ( } p=0.003 \text { ) but not } \\
\text { for the HFPS group. }\end{array}$ & $\begin{array}{l}\text { Small sample size. } \\
\text { Students in case study } \\
\text { group still received } 1 \\
\text { session of HFPS } \\
\text { which was originally in } \\
\text { the curriculum } \\
\text { potentially affecting } \\
\text { differences between } \\
\text { groups }\end{array}$ & Include \\
\hline 19 & $\begin{array}{l}\text { Shin et al. } \\
2015 \\
\text { Korea }\end{array}$ & $\begin{array}{l}\text { Students from } \\
\text { University A } \\
\text { completed one } \\
\text { simulation, } \\
\text { Students from } \\
\text { University B } \\
\text { completed two } \\
\text { simulation and C } \\
\text { three. }\end{array}$ & $\begin{array}{l}3^{\text {rd }} \text { and } 4^{\text {th }} \text { year } \\
\text { nursing students } \\
(\mathrm{n}=237) \text { across } 3 \\
\text { universities }\end{array}$ & $\begin{array}{l}\text { Yoon's Critical Thinking } \\
\text { Disposition (CTD) tool } \\
\text { completed prior to } \\
\text { intervention and on } \\
\text { completion of paediatric } \\
\text { practicum (timeframe } \\
\text { not stated) }\end{array}$ & $\begin{array}{l}\text { Students with one or two } \\
\text { exposures to simulation } \\
\text { did not demonstrate a } \\
\text { significant increase in } \\
\text { critical thinking scores. } \\
\text { Students exposed to three } \\
\text { simulations showed a } \\
\text { significant increase in } \\
\text { critical thinking scores }\end{array}$ & $\begin{array}{l}\text { Differences of teaching } \\
\text { methodology between } \\
\text { the } 3 \text { universities, with } \\
\text { one using an } \\
\text { integrated curriculum } \\
\text { and others using a } \\
\text { traditional curriculum. } \\
\text { All students had } \\
\text { experienced simulation } \\
\text { prior to intervention at } \\
\text { different degrees. CTD } \\
\text { tool was specifically } \\
\text { designed for use in } \\
\text { Korea and measured } \\
\text { disposition only. }\end{array}$ & Include \\
\hline
\end{tabular}




\begin{tabular}{|c|c|c|c|c|c|c|c|}
\hline 20 & $\begin{array}{l}\text { Evans and } \\
\text { Bendel, } \\
\text { (2004). } \\
\text { United States }\end{array}$ & $\begin{array}{l}\text { Intervention } \\
\text { Narrative } \\
\text { Pedagogy for one } \\
\text { semester. Control } \\
\text { consisted of } \\
\text { traditional } \\
\text { teaching methods } \\
\text { (not stated what } \\
\text { these were). }\end{array}$ & $\begin{array}{l}\text { Undergraduate } \\
\text { nursing students } \\
(n=114)\end{array}$ & $\begin{array}{l}\text { Used Californian Critical } \\
\text { Thinking Disposition } \\
\text { Inventory (CCTDI) pre } \\
\text { and post-test over one } \\
\text { semester. }\end{array}$ & $\begin{array}{l}\text { Statistically significant } \\
\text { improvement in CCTDI } \\
\text { scores for both groups, } \\
\text { but no significant } \\
\text { differences between } \\
\text { control and experimental } \\
\text { groups. }\end{array}$ & $\begin{array}{l}\text { Statistically significant } \\
\text { improvement for both } \\
\text { groups may indicate } \\
\text { that involvement in } \\
\text { academia increased } \\
\text { critical thinking rather } \\
\text { than the teaching } \\
\text { strategy. }\end{array}$ & Include \\
\hline \multicolumn{8}{|c|}{ Critical reading and writing course } \\
\hline 21 & $\begin{array}{l}\text { Chen, and } \\
\text { Lin, (2003) } \\
\text { Taiwan }\end{array}$ & $\begin{array}{l}\text { Intervention was a } \\
32 \text { hour course in } \\
\text { which students } \\
\text { learnt literature } \\
\text { searching, } \\
\text { critiquing and } \\
\text { academic writing. } \\
\text { It was unclear } \\
\text { what education } \\
\text { the control group } \\
\text { received. }\end{array}$ & $\begin{array}{l}\text { 1st year nursing } \\
\text { students }(n=168)\end{array}$ & $\begin{array}{l}\text { Used N3 case report } \\
\text { accreditation form. } \\
\text { Collected data using } \\
\text { student's critique of a } \\
\text { case study. Data } \\
\text { collected at baseline } \\
\text { and following completion } \\
\text { of the course. }\end{array}$ & $\begin{array}{l}\text { Experimental group } \\
\text { reported significantly } \\
\text { higher scores than control } \\
\text { group. }\end{array}$ & $\begin{array}{l}\text { Unclear whether tool } \\
\text { measured students' } \\
\text { ability to critique an } \\
\text { article rather than } \\
\text { critical thinking. } 67 \% \text { of } \\
\text { students had } \\
\text { previously written a } \\
\text { literature review and } \\
79 \% \text { had written a } \\
\text { case study which may } \\
\text { have introduced bias. } \\
\text { Improvements may be } \\
\text { accounted for by } \\
\text { repeated exposure to } \\
\text { the critique process } \\
\text { rather than thinking } \\
\text { critically. }\end{array}$ & Include \\
\hline \multicolumn{8}{|c|}{ Videotaped Vignettes } \\
\hline 22 & $\begin{array}{l}\text { Chau, et al. } \\
(2001) \text {. Hong } \\
\text { Kong }\end{array}$ & $\begin{array}{l}\text { Intervention was } 4 \\
\text { vignettes. No } \\
\text { control group was } \\
\text { used. }\end{array}$ & $\begin{array}{l}\text { 1st and } 2^{\text {nd }} \text { year } \\
\text { nursing students } \\
(n=83)\end{array}$ & $\begin{array}{l}\text { Pre-test/post-test design } \\
\text { using the Californian } \\
\text { Critical Thinking Skills } \\
\text { Test (CCTST) at } \\
\text { baseline and } 13 \text { weeks } \\
\text { following intervention. }\end{array}$ & $\begin{array}{l}\text { No statistical difference in } \\
\text { pre and post test scores. }\end{array}$ & $\begin{array}{l}\text { Students exposed to } 4 \\
\text { vignettes over } 13 \text { week } \\
\text { semester. Low dose } \\
\text { may account for the } \\
\text { minimal effect on } \\
\text { critical thinking skills. } \\
\text { No control group for } \\
\text { comparison. }\end{array}$ & Include \\
\hline
\end{tabular}




\begin{tabular}{|c|c|c|c|c|c|c|c|}
\hline 23 & $\begin{array}{l}\text { Pucer et al. } \\
(2014) \\
\text { Slovenia }\end{array}$ & $\begin{array}{l}\text { Intervention was } \\
\text { an ICT program of } \\
\text { scenarios that } \\
\text { mirror clinical } \\
\text { situations. No } \\
\text { control group. }\end{array}$ & $\begin{array}{l}1^{\text {st }} \text { year nursing } \\
\text { students }(n=40) \text {. }\end{array}$ & $\begin{array}{l}\text { Used analysis tool of pre } \\
\text { and post discussion } \\
\text { board postings. }\end{array}$ & $\begin{array}{l}\text { Qualitative analysis of the } \\
\text { discussion board posts } \\
\text { showed a significant } \\
\text { improvement in number of } \\
\text { posts }(12.2 \%) \text { for which } \\
\text { opinions and conclusions } \\
\text { of participants were } \\
\text { justified with valid } \\
\text { arguments. }\end{array}$ & $\begin{array}{l}\text { Unclear whether tool } \\
\text { measured critical } \\
\text { thinking or } \\
\text { competence in } \\
\text { discussion board } \\
\text { postings. }\end{array}$ & Include \\
\hline \multicolumn{8}{|c|}{ Web-Based Animated Pedagogical Agents } \\
\hline 24 & $\begin{array}{l}\text { Morey, } \\
\text { (2012). } \\
\text { United States }\end{array}$ & $\begin{array}{l}\text { Intervention } \\
\text { online animated } \\
\text { pedagogical } \\
\text { agent. Control } \\
\text { group received } \\
\text { traditional face to } \\
\text { face teaching. }\end{array}$ & $\begin{array}{l}\text { Final semester } \\
\text { nursing students } \\
(n=45)\end{array}$ & $\begin{array}{l}\text { Used the Critical } \\
\text { Thinking Process Test } \\
\text { (CTPT) a nursing } \\
\text { specific quantitative } \\
\text { measure and a think- } \\
\text { aloud protocol as the } \\
\text { qualitative measure. } \\
\text { Both measures } \\
\text { completed at baseline } \\
\text { and } 16 \text { weeks later. }\end{array}$ & $\begin{array}{l}\text { No differences in CT } \\
\text { levels on either tool. }\end{array}$ & $\begin{array}{l}\text { Limited information } \\
\text { regarding the think } \\
\text { aloud protocol. } \\
\text { Elements seemed to } \\
\text { relate to nursing } \\
\text { process ie collect, } \\
\text { review, relate, } \\
\text { interpret, infer, } \\
\text { diagnosis, act, and } \\
\text { evaluate, rather than } \\
\text { critical thinking }\end{array}$ & Include \\
\hline \multicolumn{8}{|c|}{ Reflective Writing } \\
\hline 25 & $\begin{array}{l}\text { Naber and } \\
\text { Wyatt, (2014) } \\
\text { United States }\end{array}$ & $\begin{array}{l}\text { Intervention group } \\
\text { completed six } \\
\text { reflective writing } \\
\text { assignments } \\
\text { Unclear what } \\
\text { education the } \\
\text { control group } \\
\text { received. }\end{array}$ & $\begin{array}{l}4^{\text {th }} \text { semester } \\
\text { nursing students ( } \mathrm{n} \\
=70) .\end{array}$ & $\begin{array}{l}\text { Californian Critical } \\
\text { Thinking Skills Test and } \\
\text { Californian Critical } \\
\text { Thinking Disposition } \\
\text { Inventory completed at } \\
\text { baseline and } 8 \text { weeks } \\
\text { later. }\end{array}$ & $\begin{array}{l}\text { Total CCTST and CCTDI } \\
\text { scores of intervention } \\
\text { group did not increase } \\
\text { significantly following the } \\
\text { intervention. No reporting } \\
\text { of reliability of CCTST or } \\
\text { CCTDI scale for this } \\
\text { study. }\end{array}$ & $\begin{array}{l}\text { Only eight weeks } \\
\text { between pre and post } \\
\text { test. Perhaps critical } \\
\text { thinking takes longer } \\
\text { than } 8 \text { weeks to } \\
\text { develop. Potential } \\
\text { contamination if } \\
\text { students discussed } \\
\text { their learning. }\end{array}$ & Include \\
\hline \multicolumn{8}{|c|}{ Grand Rounds } \\
\hline 26 & $\begin{array}{l}\text { Mann, } \\
\text { (2012). USA }\end{array}$ & $\begin{array}{l}\text { Students resolved } \\
\text { a healthcare } \\
\text { dilemma as a } \\
\text { group following a } \\
\text { simulation }\end{array}$ & $\begin{array}{l}2^{\text {nd }} \text { year nursing } \\
\text { students }(n=21) .\end{array}$ & $\begin{array}{l}\text { Assessment } \\
\text { Technologies Institute } \\
\text { (ATI) Critical Thinking } \\
\text { Assessment (CTA) at } \\
\text { commencement of }\end{array}$ & $\begin{array}{l}\text { No significant difference } \\
\text { between CT scores for } \\
\text { the two groups. Students } \\
\text { in control group reported }\end{array}$ & $\begin{array}{l}\text { The educational } \\
\text { intervention was not } \\
\text { clearly described. Very } \\
\text { small sample size. As } \\
\text { critical thinking initially }\end{array}$ & Include \\
\hline
\end{tabular}




\begin{tabular}{|c|c|c|c|c|c|c|c|}
\hline & & $\begin{array}{l}\text { technique. The } \\
\text { education } \\
\text { strategy appears } \\
\text { to be grand } \\
\text { rounds but not } \\
\text { specified. Control } \\
\text { condition not } \\
\text { specified. }\end{array}$ & & $\begin{array}{l}\text { program and at } \\
\text { completion of course. }\end{array}$ & $\begin{array}{l}\text { decreased critical thinking } \\
\text { ability. }\end{array}$ & $\begin{array}{l}\text { measured at beginning } \\
\text { of program rather than } \\
\text { immediately prior to } \\
\text { the intervention other } \\
\text { variables/factors could } \\
\text { have affected critical } \\
\text { thinking skills. }\end{array}$ & \\
\hline \multicolumn{8}{|c|}{ Interactive Videodisc System (IVS) } \\
\hline 27 & $\begin{array}{l}\text { Yeh and } \\
\text { Chen (2005). } \\
\text { Taiwan }\end{array}$ & $\begin{array}{l}\text { Educational } \\
\text { intervention - } 2 \\
\text { hour lecture on } \\
\text { critical thinking } \\
\text { and an optional } \\
\text { IVS program. }\end{array}$ & $\begin{array}{l}\text { RN-BN students } \\
(n=126) \text { enrolled in } \\
\text { a medical-surgical } \\
\text { course. }\end{array}$ & $\begin{array}{l}\text { Californian Critical } \\
\text { Thinking Disposition } \\
\text { Inventory (CCTDI) at } \\
\text { baseline and } 6 \text { weeks } \\
\text { later following the } \\
\text { course. }\end{array}$ & $\begin{array}{l}\text { Statistically significant } \\
\text { differences between pre } \\
\text { and post-test overall } \\
\text { scores. }\end{array}$ & $\begin{array}{l}\text { Only measured critical } \\
\text { thinking disposition } \\
\text { rather than CT skills. } \\
\text { IVS participation } \\
\text { ranged from } 15 \text { to } 150 \\
\text { minutes. Did not } \\
\text { correlate changes in } \\
\text { CCTDI with time spent } \\
\text { on IVS. }\end{array}$ & Include \\
\hline \multicolumn{8}{|c|}{ Evidence-Based Nursing Education Course } \\
\hline 28 & $\begin{array}{l}\text { Zadeh et al. } \\
\text { (2014). Iran }\end{array}$ & $\begin{array}{l}\text { Intervention } \\
\text { research } \\
\text { methodology and } \\
\text { evidence based } \\
\text { nursing course. } \\
\text { Control condition } \\
\text { not described. }\end{array}$ & $\begin{array}{l}\text { 3rd year nursing } \\
\text { students }(n=48) \text {. }\end{array}$ & $\begin{array}{l}\text { Used Californian Critical } \\
\text { Thinking Disposition } \\
\text { Inventory (CCTDI) prior } \\
\text { to intervention and one } \\
\text { month later. }\end{array}$ & $\begin{array}{l}\text { CCTDI scores were } \\
\text { significantly higher in } \\
\text { intervention group. }\end{array}$ & $\begin{array}{l}\text { Low pre and post test } \\
\text { scores (means of } 26 \\
\text { and } 36 \text { ). Relevance to } \\
\text { other countries may be } \\
\text { limited. }\end{array}$ & Include \\
\hline \multicolumn{8}{|c|}{ Peer Active Learning Strategies Approach (PALS) } \\
\hline 29 & $\begin{array}{l}\text { Stevens et } \\
\text { al. (2009) } \\
\text { USA }\end{array}$ & $\begin{array}{l}\text { Peer active } \\
\text { learning } \\
\text { strategies } \\
\text { approach (PALS). } \\
\text { No control group. }\end{array}$ & $\begin{array}{l}\text { Undergraduate } \\
\text { nursing students } \\
(n=15)\end{array}$ & $\begin{array}{l}\text { Critical Thinking Likert } \\
\text { Scale (CTLS) completed } \\
\text { prior to and following } \\
\text { clinical experience (no } \\
\text { timeframe given). }\end{array}$ & $\begin{array}{l}\text { Increase in scores on } \\
\text { CTLS but no statistical } \\
\text { analysis performed. No } \\
\text { reporting of reliability of } \\
\text { CTLS for this study or } \\
\text { previously. }\end{array}$ & $\begin{array}{l}\text { No information } \\
\text { provided on } \\
\text { recruitment of sample. } \\
\text { No validity or reliability } \\
\text { testing of tool. Very } \\
\text { small sample size. }\end{array}$ & $\begin{array}{l}\text { Exclude. No } \\
\text { statistical } \\
\text { analysis. } \\
\text { Descriptive } \\
\text { design. Tool } \\
\text { validity and } \\
\text { reliability } \\
\text { unknown. }\end{array}$ \\
\hline
\end{tabular}




\section{Teaching strategies}

\section{Problem Based Learning}

Seven studies measured the effects of problem based learning (PBL) on the development of critical thinking (Choi, et al., 2014; Tiwari, et al., 2006; Jones, 2008; Jun, et al., 2013; Yu, et al., 2012; Yuan et al., 2008; Dehkordi and Heydarnejad, 2008). Two studies measured the effect of both PBL and concept mapping on critical thinking (Tseng et al., 2011; Orique et al., 2015). All but one study (Choi et al, 2014) found PBL to have a positive effect with an increase in critical thinking scores. Three studies that tested a PBL intervention used the CCTDI to measure critical thinking change. In a 2-year longitudinal study students in the intervention group experienced PBL for an academic year (Tiwari et al., 2006). Students receiving the intervention had significantly higher overall CCTDI scores which they attributed to PBL. Similar results were obtained by Yu et al. (2012) and Dehkordi and Heydarnejad (2008) who also used the CCTDI to measure the effects of PBL on critical thinking. However, Yu et al. (2012) found no differences in the subscale scores related to analyticity, systematicity, and self-confidence. The conflicting results between these studies using the same intervention and tool may indicate a lack of sensitivity by the CCTDI to measure critical thinking change in nursing practice. The CCTDI relies on self-report which may be affected by social response bias (Tiwari, et al, 2006). Furthermore, the CCTDI measures student's critical thinking disposition, or the self-perceived likelihood of them thinking critically, whereas critical thinking is considered to have two dimensions; disposition and skills (Facione, 1990). The increase in critical thinking disposition may not translate into the application of critical thinking skills in nursing practice.

Seven of the nine studies involving PBL were conducted in Asian and Middle Eastern countries (Korea, Hong Kong, Taiwan, China and Iran), where cultural influences may adversely affect critical thinking development of students. According to the recommended minimum cut-off scores on the CCTDI, scores between 30 and 40 indicates "weakness to ambivalence" towards that critical thinking domain (Facione and Facione, 1992). In two of the three PBL studies using the CCTDI (Tiwari, et al., 2006; Yu, et al.,2012), domain scores did not reach the cut-off score of 40 in either pre or post-tests, indicating a weak critical thinking disposition. This may be indicative of cultural influences in those Asian countries. Predominant characteristics of learning environments in Asia such as adherence to didactic models of teaching, dominance of the medical model, and not questioning those in authority, do not foster independent thinking (Lim et al., 2009; Chan et al., 2011). Thus, the applicability of results from these studies to other cultures where students are encouraged to 
question and think independently may be limited. Nevertheless, these studies do provide a baseline from which future studies in those countries may demonstrate general improvement.

The impact of culture on different learning environments may also influence teaching approaches. In four of the nine studies related to PBL students had previously only been exposed to didactic teaching methods (Dehkordi and Heydarnejad, 2008; Jun et al., 2013; Tiwari et al., 2006; Yuan, et al., 2008). Didactic teaching promotes rote learning and offers little opportunity for students to question and consider the application of nursing knowledge to practice. Although results from four studies were favourable, PBL was the only active learning approach students had experienced. The increase in critical thinking scores may simply reflect encouragement by teachers for students to think actively.

Critical thinking skills were found to improve in the intervention group following PBL, measured through grading of developed nursing care plans (Jones, 2008). However, it was not clear whether the tool used to grade the care plans was validated by experts or if the items measured dimensions of critical thinking. Methodological rigour is questionable as the principal investigator taught both groups, and graded the care plans, introducing potential bias.

The use of both PBL and concept mapping on critical thinking was examined in two studies (Tseng et al., 2011; Orique et al., 2015). Students receiving PBL and concept mapping reported significantly higher Critical Thinking Scale (CTS) scores upon completion of course and 6 months later (Tseng et al., 2011). There was limited information regarding the CTS tool and how it measured critical thinking. However, promising follow-up results suggested that PBL was an effective long term strategy to increase critical thinking in this cohort. As this study was conducted in Taiwan, baseline levels of critical thinking may have been low, small changes may have been significant, and culture may have contributed to positive results.

Orique et al. (2015) measured the effects of PBL and concept mapping separately on critical thinking using the Holistic Critical Thinking Rubric (HCTR). Students submitted nursing care plans prior to and following, PBL instruction and concept mapping sessions. The significant increase in critical thinking scores across time for these first year students could have related to students' developing care planning expertise rather than improved cognitive skills. The assessment process was not well described; there was no detail on whether assessment was undertaken by a lecturer who was blind to the intervention; and if teachers' ratings of students work were moderated. 
The effect of PBL on critical thinking development appears favourable, with seven of the nine studies reporting positive outcomes. However, due to the use of different measurement tools (some of which had not been validated), different cultural influences on critical thinking development and learning approaches, and lack of methodological rigour, these results need to be viewed with caution. None of the included studies provided sufficient descriptions of PBL processes to ensure fidelity and enable replication by other researchers. There was no information on the preparation of staff to teach PBL methods and no quality review of classroom processes reported such as the extent to which students were encouraged to question, the extent to which teachers facilitated rather than lead learning; ability of students to work together to generate new knowledge, quality of the case studies and quality of supplementary resource materials for each case.

\section{Concept mapping}

Four studies measured the effect of concept mapping on critical thinking. Three studies (Atay and Karabacak, 2012; Lee et al., 2013; Abel and Freeze, 2006) found an increase in CT scores following concept mapping education and activities. The effects of preparing care plans using concept maps on critical thinking was measured using the CCTDI (Atay and Karabacak, 2012). Students receiving the intervention had statistically significant higher critical thinking scores and mean scores on concept map care plan evaluation criteria. However, there were inconsistencies in the length of the educational intervention, with the intervention group receiving an extra nine to twelve hours of education specifically on concept mapping. The increase in critical thinking scores could have been a result of extra educational hours rather than as a direct result of the concept mapping education.

A two year longitudinal study tested the effects of concept map education on critical thinking skills using the Critical Thinking Scale (CTS) at four time-points. The CTS measures critical thinking through assessment of inference, recognition of assumptions, deduction, interpretation, and evaluation of argument concepts (Lee et al., 2013). The 15 week course on concept mapping produced an initial improvement in critical thinking scores but this decreased over time for all students. The results may indicate that the intervention was not integrated or impactful as students experienced didactic teaching methods for the remainder of the program.

Another longitudinal study evaluated the effects of concept mapping with 28 associate degree nursing students (Abel and Freeze, 2006). A validated concept map scoring criteria was used to measure critical thinking development at four time-points over a one year period (Abel and Freeze, 2006). The authors demonstrated increases in students' concept map scores as they progressed through the curriculum. However, it was unclear how the scoring criteria related to critical thinking 
and whether increased scores were a true reflection of improved critical thinking or simply improved competence in concept mapping.

Wheeler and Collins (2003) did not demonstrate the same effect in their 7.5 week study. Pre and post test scores on the California Critical Thinking Skills Test (CCTST) of students taught concept mapping compared to a control group who received instruction on preparing traditional nursing care plans did not differ. Increased critical thinking scores were discerned in both groups, with no difference between groups. This suggests that both teaching approaches were effective in increasing critical thinking development.

\section{Simulation}

Six studies examining the effects of simulation on development of critical thinking had variable findings. Two studies reported increased critical thinking scores following the intervention (SullivanMann et al., 2009; Wood and Toronto 2012). Shin et al., (2015) found the increase in critical thinking was dose dependent, with increases in scores occurring with three simulations but not with two or one. Another study demonstrated no increase in critical thinking scores, and some decreases (Shinnick and Woo 2013), while another study found higher scores in the control group (Goodstone et al., 2013). The final study which used two outcome measures reported contradictory findings between tools (Ravert, 2008).

The effect of multiple simulations on critical thinking over a period of six weeks was measured using the HSRT (Sullivan-Mann et al.,2009). Groups were exposed to two (control) and five (intervention) clinical simulations. Although both groups had increased scores on the post-test, the intervention group had statistically higher scores. The authors suggested results may have been affected by the experience of the clinical facilitator (Sullivan-Mann et al., 2009).

Another study tested a brief intervention involving a single human patient simulation with a two week lapse between pre and post-test using the HSRT (Shinnick and Woo, 2013). There was a decrease in critical thinking scores, perhaps related to response burden. The HSRT takes 40-50 minutes and asking students to complete this tool twice in two weeks may have been time intensive particularly in response to a low-dose intervention. In contrast, a brief two hour human patient simulation intervention with the same two week timeframe between testing resulted in higher mean post-test scores for the experimental group using the CCTDI (Wood and Toronto, 2012). The CCTDI takes around 20-30 minutes to complete. As the HSRT measures the application of critical thinking and the CCTDI measures student's disposition for critical thinking, differences in results in these two 
studies could suggest that a short intervention may influence a change in students' tendency to think critically but does not change their ability to apply critical thinking skills.

To test the effect of simulations on critical thinking Shin et al. (2015) used a multisite approach where one site offered one simulation, another offered two simulations and the third site conducted three simulations. Improved critical thinking scores were only noted for the students who participated in three simulations. It was not clear whether students who participated in fewer sessions still received teaching for the same length of time. Results could also have been affected by different teaching approaches at the three universities with one using an integrated curriculum and the other two using a traditional one. A potential limitation of the study was that the Critical Thinking Disposition (CTD) tool measures students' disposition whereas simulation requires the practical application of critical thinking skills. The CTD was specifically designed for use in Korea and items may not be applicable to education in other countries.

Ravert (2008) used the CCTST and CCTDI to measure critical thinking development in undergraduate nursing students allocated into simulation ( 5 hours), non-simulation (5 hours) and control groups. The control group demonstrated higher scores on the CCTDI compared to non- simulation and simulation groups whereas the two intervention groups scored higher on the CCTST. Differences in scores did not reach statistical significance as the small sample $(n=30)$ size may have been underpowered to detect group differences.

Contradictory results were also found in a study comparing the effect of high fidelity patient simulation to case study teaching (Goodstone et al., 2013). The post-test HSRT administered 14 weeks later revealed significantly higher scores for the case study group compared to the high fidelity patient simulation group. Results may have been affected by the small sample size $(n=42)$, and possible contamination as the case study group experienced one simulation session as well.

Contradictory findings were demonstrated by the five studies measuring the effect of simulation on critical thinking. Three studies utilised the HSRT and found inconsistent results. The effect of simulation on critical thinking is uncertain and may have been affected by the relatively short intervention dose and short timeframe between pre and post-testing. Critical thinking is considered by some to be an 'ingrained' trait (Ravert, 2008) and take significant time to change or develop (Choi, 2014). Thus a short intervention may not be adequate to impact on critical thinking abilities.

\section{Narrative Pedagogy}


The effect of narrative pedagogy on critical thinking development was examined over one semester using the CCTDI (Evans and Bendel, 2004). Critical thinking scores were expected to increase based on results of a five year project on narrative pedagogy which demonstrated improved integration of theory with practice, and empowerment as clinicians (Severtsen and Evans 2000). However, although critical thinking scores improved for both groups, no statistical differences between the control and experimental groups were noted. It could be that critical thinking disposition increases in an excellent academic environment regardless of the teaching strategy.

\section{Critical reading and writing course}

The N3 case report accreditation form developed by the Taiwan Nurses Association was used to assess students' critical thinking abilities in the critique of case study reports (Chen and Lin, 2003). Students in the intervention group received education on the process of literature searching, critical reading and writing. Students in the experimental group had significantly higher case study scores than the control group. Improved scores by the experimental group may be accounted for by their repeated exposure to the critique process and may reflect their improved ability to critique an article rather than think critically.

\section{Videotaped vignettes}

The effect of videotaped vignettes on critical thinking skills for $1^{\text {st }}$ and $2^{\text {nd }}$ year students was measured using the CCTST (Chau et al., 2001). There was no control group and no statistical difference was found between pre and post test scores. Although the course was conducted over 13 weeks, students were only exposed to 4 vignettes in this period. Intervention dose may have been insufficient to develop students' capacity to address problems that were new to them (Chau, et al., 2001).

\section{ICT based modern approach}

Pucer et al. (2014) used a newly developed discussion board analysis tool to identified core key elements of critical thinking as defined by Facione (1990). A significant improvement in the percentage of posts where students' opinions and conclusions were justified with valid arguments was reported (Pucer et al., 2014). However, there was limited information on development of the tool, process of expert review and validation, or inter-rater reliability. It was unclear whether the tool measured student's critical thinking abilities or their competence in discussion board postings.

Web-based animated pedagogical agents 
Both a newly developed qualitative tool using a think aloud protocol, and a standardised tool named the Critical Thinking Process Test (CTPT) were used to measure the effects of an animated pedagogical agent on critical thinking (Morey, 2012). Results differed according to the tool used. Although both groups improved there were no significant differences on CTPT scores and correct conclusions using the think-aloud protocol. The pedagogical agent group had significantly better results on the cognitive process of evaluation. These mixed results may indicate the difficulty in measuring critical thinking development in an exam context.

\section{Reflective writing intervention}

An eight week reflective writing intervention was used with 70 fourth semester students randomised into control and intervention groups (Naber and Wyatt, 2014). No statistically significant increases in critical thinking scores using the CCTDI and CCTST were demonstrated in the intervention group and there were no differences between groups.

\section{Grand rounds}

The effectiveness of a 'grand round' education strategy on critical thinking was assessed using the CTA (Mann, 2012). No statistical differences between intervention and control groups were found however the intervention group improved more. The intervention group received more hours of education and instruction from the researcher which could have affected results. Also the pre-test was conducted at the beginning of the program rather than immediately prior to the intervention so results may have been confounded by variables not considered in this study.

Interactive videodisc systems (IVS)

A brief intervention consisting of a two hour lecture and an optional interactive videodisc system (IVS) produced increased scores on the CCTDI with 6 weeks between pre and post-test (Yeh and Chen, 2005). However, student participation in the interactive videodisc system ranged from five to 150 minutes. It would have been useful to correlate changed CCTDI scores with duration of participation with the interactive videodisc system. The increased scores may have also been affected by cultural influences as four of the seven pre-test domain scores and one post-test domain did not reach the minimum cut-off score of 40 , indicating weak baseline critical thinking disposition in students.

\section{Evidence based course}

A study in Iran examined the effects of an evidenced based nursing course reported improved CCTDI scores (Zadeh et al., 2014). However, the low mean pre and post-test scores on the CCTDI (means of 
26 and 36 retrospectively), indicated improved, but still weak critical thinking disposition in students. This may relate to Iranian culture where nursing education methods concentrate on memorisation of facts. Prior to this course students had only been exposed to didactic teaching methods (Zadeh et al., 2014). The relevance of these findings is limited in other countries where evidence based education is embedded within curricula.

\section{Discussion}

This review included 28 studies from 8 different countries testing 12 different teaching interventions to promote the development of critical thinking. None of the included studies involved midwifery students and highlights a significant gap in midwifery education literature. The findings of the review also need to be considered in light of limitations associated with methodological rigour, cultural influences, appropriateness of the measurement tool, duration of intervention, timing of pre and post-testing, and intervention versus control dose.

Results of included studies varied, with little consistency across studies using the same type of intervention or outcome tool. Seventeen studies identified a significant increase in CT of nursing students following an educational intervention, while nine reported no increases and some found unexplained decreases in critical thinking. In four studies the education intervention was longer than the control condition (Ravert, 2008; Atay and Karabacak, 2012; Wood and Toronto, 2012; Mann, 2012). These discrepancies in 'dose' across conditions may have biased results.

Critical thinking in these nursing studies was often measured following a single brief intervention (Shinnick and Woo, 2013; Wood and Toronto, 2013; Sullivan-Mann et al., 2009; Ravert, 2008; Yeh and Chen, 2005) with limited success. Given that critical thinking is considered to develop over time (Paul, 1993; Choi et al., 2014), the length of an intervention is an important educational and research consideration. Other methodological weaknesses related to the timing of pre and post-test measurement which ranged from 2 weeks to 4 years. A short testing interval may not only result in survey fatigue but only reflect short-term improvement of critical thinking due to new learning. Sustained longer term effects were rarely assessed. Interventions are more likely to successfully develop critical thinking if offered over an extended period of time, there is a progressive scaffolding of skills; educators are competent to offer the intervention, and interventions are integrated throughout the curriculum.

Promising results were found on the effects of PBL and concept mapping on critical thinking development. This is not surprising given the constructivist principles underpinning these teaching 
methodologies. PBL and concept mapping challenge students to actively participate in building knowledge from what is known towards a new understanding (Piaget, 1977; Vygotsky, 1986). Although the construction of new knowledge commonly involves engagement in research activities, and the use of intelligence and reasoning, the co-operative and interactive nature of problem based learning fosters students' critical thinking in relation to clinical problems.

Variable results were found on critical thinking development when using simulation. Inconsistencies in the intervention dose and small samples sizes could account from some of this variation. However, using simulation as a teaching strategy to improve critical thinking requires further investigation. According to Mong-Chue (2000) critical thinking involves controlled, purposeful and conscious thought processes. Although simulation activities can be useful in developing clinical skills it is uncertain whether they develop critical thinking skills which involves the interpretation of multiple data sources (Mitchell et al., 2009). Within clinical simulation students are often required to make rapid decisions, critical thinking requires a deeper learning methodology using analytical skills and analytical skills and acumens beyond this (Carter et al., 2014).

Positive and promising results were found with a small number of studies using other interventions including; the use of discussion boards, critical reading and writing courses and narrative pedagogy, which require further investigation. Positive findings related to these interventions could be due to the use of active constructivist-based learning strategies.

The increases in critical thinking performance can be understood more readily by exploring cognitive development theories by researchers such as Piaget (1977) and Vygotsky (1986). When students engage in a clinical problem which cannot be easily resolved cognitive dissonance occurs. Active learning strategies such as PBL, concept mapping and simulation framed around clinical scenarios, enable students to further construct their knowledge in relation to the concepts in question. Constructivist learning is enhanced by using experiential learning methods (such as simulation) and peer interaction (during PBL) which promotes cognitive development because of discussion around critical cognitive conflicts. Given that constructivists see learners as constructing their own knowledge, more attention also needs to be paid to learning from experience (Boud \& Edwards, 1999).

Some results of this review may have been influenced by possible cultural influences. Seven studies using PBL and one testing concept mapping were conducted in Asian and Middle Eastern countries where didactic methods are the norm. Passive learning through lectures is well known to limit 
critical thinking development as it focusses on the memorisation of content (Diekelmann and Smythe, 2004; Ironside, 2004). It could be that any type of active teaching strategy will increase critical thinking disposition and skill scores in these circumstances. The evaluation of similar strategies in countries where a diverse range of teaching methods are used may not achieve the same level of change in critical thinking development.

Educational and practice contexts in countries such as the United Kingdom, United States and Australia reflect a wide variety of active teaching and assessment strategies. Increasingly, midwifery and nursing programs in Australia involve blended and online learning. Rather than using one specific teaching or learning strategy, a scaffolded approach of active learning and authentic assessment, including clinical assessment is used (Carter et al., 2014). Assessing the development of critical thinking in these programs would require longitudinal, multi-method measurement. Many studies in the review used brief teaching and learning interventions which had limited impact on nursing students' critical thinking. Raymond-Seniuk and Profetto-McGrath (2011) suggest pluralism or multiple lenses are also needed to capture the depth and breadth of the knowledge and essence of midwifery and nursing practice. The use of multiple outcome measures and triangulation of data may provide greater insight into the effectiveness of teaching methods on critical thinking. Such approaches may also contribute to the development of critical thinking methodologies specifically in nursing and midwifery. Understanding and testing the theories underpinning different teaching interventions is needed in order to continue advance our knowledge in this field.

The review itself was limited to empirical studies published in English that used measures of critical thinking with midwifery and nursing students. It could be that these criteria unnecessarily restricted the scope of the review. Future reviews could consider an evaluation of teaching interventions to promote critical thinking across health professional groups. Given the high proportion of researchers using the California Critical Thinking Disposition Inventory (CCTDI) (8 studies), the California Critical Thinking Skills Test (CCTST) (3 studies), future reviews could also consider a meta-analysis of results from these tools across a broad range of health professional students. However, it could also be argued that the continued use of generalised critical thinking tools is unlikely to help identify appropriate teaching methods to improve critical thinking abilities of nursing and midwifery students. Discipline specific strategies and tools that measure the student's ability to apply critical thinking in practice are needed.

\section{Conclusion}


Common educational interventions used to promote critical thinking development were PBL, simulation, and concept mapping. There were methodological concerns about most studies such as small sample size and a lack of quality assurance on the delivery of an intervention. The short duration of interventions did not allow sufficient time for students to develop critical thinking skills. Variability in the timing between pre and post-tests was evident, with the majority of studies repeating the outcome measures soon after the completion of the intervention. Longer term effects of interventions on critical thinking were rarely reported. There were also inconsistencies in intervention and control doses and in some studies, the control group experienced smaller doses, introducing potential bias. In some studies, these factors were confounded by cultural influences on critical thinking development.

Academics need to continue to strive to maximise student's critical thinking abilities, preparing them to be competent, effective and autonomous nursing and midwifery graduates. Whilst this review aimed to identify effective teaching strategies that promote and develop critical thinking, flaws in methodology and outcome measures contributed to inconsistent findings. 


\section{References}

Abel, W.M., \& Freeze, M., 2006. Evaluation of concept mapping in an associate degree nursing program. Journal of Nursing Education, 45(9), 356-364.

Atay, S., \& Karabacak, Ü., 2012. Care plans using concept maps and their effects on the critical thinking dispositions of nursing students. International Journal of Nursing Practice, 18(3), 233-239. doi: 10.1111/j.1440-172X.2012.02034.x.

Banfield, V., Fagan, B., \& Janes, C. 2012. Charting a new course in knowledge: Creating life-long critical care thinkers. Dynamics, 23(1), 24-18.

Boud, D. \& Edwards, H. (1999). Learning for practice: Promoting learning in clinical and community settings. In J. Higgs \& H. Edwards (Eds.), Educating beginning practitioners: Challenges for health professional education (pp. 173-179). Oxford: Butterworth-Heinemann.

Brunt, B.A., 2005. Models, measurement, and strategies in developing critical-thinking skills. Journal of Continuing Education in Nursing, 36(6), 255-262.

CASP, 2013. Critical Thinking Appraisal Skills Programme:CASP Checklists. UK: CASP. Retrieved from http://www.casp-uk.net/\#!casp-tools-checklists/c18f8

Carter, A.G., Sidebotham, M., Creedy, D.K., Fenwick, J., \& Gamble, J., 2014. Using root cause analysis to promote critical thinking in final year Bachelor of Midwifery students. Nurse Education Today, 34(6), 1018-1023. doi: 10.1016/j.nedt.2013.10.020

Carter, A.G., Creedy, D.K. \& Sidebotham, M., 2015 Evaluation of tools used to measure critical thinking development in nursing and midwifery undergraduate students: A systematic review. Nurse Education Today. 35(7):864-874. doi: 10.1016/j.nedt.2015.02.023.

Castledine, G., 2010. Critical thinking is crucial. The British Journal of Nursing. 19 (4), 271.

Chan, Z.C.Y., 2013. A systematic review of critical thinking in nursing education. Nurse Education Today, 33(3), 236-240. doi: 10.1016/j.nedt.2013.01.007.

Chan, M.F., Creedy, D.K., Chua, T.L., \& Lim, C.C. 2011. Exploring the psychological health related profile of nursing students in Singapore: a cluster analysis. Journal of Clinical Nursing, 20 (2324): 3553-3560.

Chau, J.P.C., Chang, A.M., Lee,I.F.K., Ip,W.Y., Lee, D.T.F., \& Wootton, Y., 2001. Effects of using vidoetaped vignettes on enhancing students' critical thinking ability in a baccalaureate nursing programme. Journal of Advanced Nursing, 36(1), 112-119. doi: 10.1046/j.13652648.2001.01948.x

Chen, F., \& Lin, M., 2003. Effects of a nursing literature reading course on promoting critical thinking in two-year nursing program students. Journal of Nursing Research (Taiwan Nurses Association), 11(2), 137-147.

Choi, E., Lindquist, R., \& Song, Y., 2014. Effects of problem-based learning vs. traditional lecture on Korean nursing students' critical thinking, problem-solving, and self-directed learning. Nurse Education Today, 34(1), 52-56. doi: 10.1016/j.nedt.2013.02.012.

Dehkordi, A.H., \& Heydarnejad, M.S., 2008. The effects of problem-based learning and lecturing on the development of Iranian nursing students' critical thinking. Pakistan Journal of Medical Sciences, 24(5), 740-743.

Diekelmann, N., \& Smythe, E., 2004. Covering content and the additive curriculum: how can I use my time with students to best help them learn what they need to know? Journal of Nursing Education, 43 (8), 341-344.

Evans, B.C., \& Bendel, R., 2004. Cognitive and ethical maturity in baccalaureate nursing students: did a class using narrative pedagogy make a difference? Nursing Education Perspectives, 25(4), 188-195.

Facione, P. A., 1990. Critical Thinking: A Statement of Expert Consensus for Purposes of Educational Assessment and Instruction, Executive Summary: "The Delphi Report". CA: The Californian Academic Press. Retrieved from http://assessment.aas.duke.edu/documents/Delphi_Report.pdf 
Facione, P. A. \& Facione, N. C., 1992. The California Critical Thinking Dispositions Inventory (CCTDI); and the CCTDI Test manual. Millbrae, CA: California Academic Press.

Goodstone, L., Goodstone, M.S., Cino, K., Glaser, C.A., Kupferman, K., \& Dember-Neal, T., 2013. Effect of simulation on the development of critical thinking in associate degree nursing students. Nursing Education Perspectives, 34(3), 159-162.

Ironside, P., 2004. Covering content and teaching thinking: Deconstructing the additive curriculum. Journal of Nursing Education, 43, 5-12.

Jones, M., 2008. Developing clinically savvy nursing students: an evaluation of problem-based learning in an associate degree program. Nursing Education Perspectives, 29(5), 278-283.

Kable, A.K., Pich, J., \& Maslin-Prothero, S.E., 2012. A structured approach to documenting a search strategy for publication: A 12 step guideline for authors. Nurse Education Today, 32, 878886.

Jun, W.H., Lee, E.J., Park, H.J., Chang, A.K., \& Kim, M.J., 2013. Use of the $5 E$ learning cycle model combined with problem-based learning for a fundamentals of nursing course. Journal of Nursing Education, 52(12), 681-689.

Lee, W., Chiang, C.H., Liao, I.C., Lee, M.L., Chen, S.L., \& Liang, T., 2013. The longitudinal effect of concept map teaching on critical thinking of nursing students. Nurse Education Today, 33(10), 1219-1223. doi: 10.1016/j.nedt.2012.06.010.

Lim, C.C., Chua, T.L., Creedy, D.K. \& Chan, M.F., 2009. A preliminary study of stress and coping in undergraduate nursing students in Singapore. Asia Pacific Psychiatry, 1, 74-80

Mann, J., 2012. Critical thinking and clinical judgment skill development in baccalaureate nursing students. Kansas Nurse, 87(1), 26-31.

Mitchell, M., Henderson, A., Groves, M., Nultey, D., 2009. The objective structured clinical examination (OSCE): optimising its value in the undergraduate nursing curriculum. Nurse Education Today 29, 398-404.

Mong-Chue, C., 2000. The challenges of midwifery practice for critical thinking. British Journal of Midwifery, 8(3), 179-183.

Morey, D.J., 2012. Development and evaluation of web-based animated pedagogical agents for facilitating critical thinking in nursing. Nursing Education Perspectives, 33(2), 116-120. doi: 10.5480/1536-5026-33.2.116.

Muoni, T., 2012. Decision-making, intuition, and the midwife: Understanding heuristics. British Journal of Midwifery, 20(1), 52-56.

Naber, J., \& Wyatt, T.H., 2014. The effect of reflective writing interventions on the critical thinking skills and dispositions of baccalaureate nursing students. Nurse Education Today, 34(1), 6772. doi: 10.1016/j.nedt.2013.04.002

Paul, R.W.(1993). Critical thinking: what every person needs to survive in a rapidly changing world. (2nd.ed) Santa Rosa, CA: Foundation for Critical Thinking.

Piaget, J. (1977). The moral judgement of the child. Harmondsworth: Penguin.

Popil, I., 2011. Promotion of critical thinking by using case studies as teaching method. Nurse Education Today, 31, 204-207.

Pucer, P., Trobec, I., \& Žvanut, B., 2014. An information communication technology based approach for the acquisition of critical thinking skills. Nurse Education Today, 34(6), 964-970. doi: 10.1016/j.nedt.2014.01.011

Ravert, P., 2008. Patient simulator sessions and critical thinking. Journal of Nursing Education, 47(12), 557-562. doi: 10.3928/01484834-20081201-06

Raymond-Seniuk, C., \& Profetto-McGrath, J., 2011. Can one learn to think critically? - A philosophical exploration. Open Nursing Journal, 5, 45-51.

Scheffer, B.K., \& Rubenfeld, M.G., 2000. A consensus statement on critical thinking in nursing. Journal of Nursing Education, 39(8), 352-359.

Severtsen, B. M., \& Evans, B.C., 2000. Education for caring practice. Nursing and Health Care Perspectives, 21(4), 172-177. 
Shin, H., Ma, H., Park, J., Ji, E.S., \& Kim, D.H., 2015. The effect of simulation courseware on critical thinking in undergraduate nursing students: Multi-site pre-post study. Nurse Education Today, 35(4), 537-542. doi: http://dx.doi.org/10.1016/j.nedt.2014.12.004.

Shinnick, M.A., \& Woo, M.A., 2013. The effect of human patient simulation on critical thinking and its predictors in prelicensure nursing students. Nurse Education Today, 33(9), 1062-1067. doi: 10.1016/j.nedt.2012.04.004

Stevens, J., Brenner, C., \& Brenner, Z.R., 2009. The peer active learning approach for clinical education: a pilot study. Journal of Theory Construction \& Testing, 13(2), 51-56.

Sullivan-Mann, J., Perron, C.A., \& Fellner, A.N., 2009. The effects of simulation on nursing students' critical thinking scores: a quantitative study. Newborn and Infant Nursing Reviews, 9(2), 111 116.

Tiwari, A., Lai, P., So, M., \& Yuen, K., 2006. A comparison of the effects of problem-based learning and lecturing on the development of students' critical thinking. Medical Education, 40(6), 547-554.

Tseng, H.C., Chou, F.H., Wang, H.H., Ko, H.K., Jian, S.Y., \& Weng, W.C., 2011. The effectiveness of problem-based learning and concept mapping among Taiwanese registered nursing students. Nurse Education Today, 31(8), e41-46. doi: 10.1016/j.nedt.2010.11.020

Vygotsky, L. (1986). Thought and language. Cambridge, MA: MIT Press.

Wheeler, L. A., \& Collins, S.K.R., 2003. The influence of concept mapping on critical thinking in baccalaureate nursing students. Journal of Professional Nursing, 19(6), 339-346.

Wood, R.Y., \& Toronto, C.E., 2012. Measuring critical thinking dispositions of novice nursing students using human patient simulators. Journal of Nursing Education, 51(6), 349-352. doi: 10.3928/01484834-20120427-05

Yeh, M., \& Chen, H., 2005. Effects of an educational program with interactive videodisc systems in improving critical thinking dispositions for RN-BSN students in Taiwan. International Journal of Nursing Studies, 42(3), 333-340.

Yu, D., Zhang, Y., Xu, Y., Wu, J., \& Wang, C., 2012. Improvement in critical thinking dispositions of undergraduate nursing students through problem-based learning: a crossover-experimental study. Journal of Nursing Education, 52(10), 574-581.

Yuan, H., Kunaviktikul, W., Klunklin, A., \& Williams, B.A., 2008. Improvement of nursing students' critical thinking skills through problem-based learning in the People's Republic of China: a quasi-experimental study. Nursing \& Health Sciences, 10(1), 70-76.

Zadeh, H.H., Khajeali, N., Khalkhali, H., \& Mohammadpour, Y., 2014. Effect of evidence-based nursing on critical thinking disposition among nursing students. Life Science Journal, 11 (9 Spec. Issue), 487-491. 